instant feedback about their current health status; 2) Personalized advice: patients want a system that can adapt medication, sport activities and food recommendations to their current health status; 3) Transparency: patients want to know the source, reasons, and individualized interpretation of any recommended changes to the management of their condition. Additionally, patients have a desire to adapt their lifestyle to the needs of $\mathrm{HF}$, but they require help to remain motivated to achieve this goal.

Conclusion These findings provide valuable information for the development and implementation of eHealth solutions. Patients want reassurance $24 / 7$, independently of the availability of healthcare services, combined with personalized medical advice regarding day-to-day management of their HF. In a next step, we are planning a multicentre clinical trial to test the first prototype of the eHealth product (DoctorMe).

\section{DYSTROPHIN DEFICIENT CARDIOMYOPATHY - PREDICTORS ASSOCIATED WITH THE CARDIAC PHENOTYPE IN A DUCHENNE'S REGISTRY POPULATION - A GUIDE FOR DEVICE THERAPY}

1J Carron, ${ }^{1} \mathrm{~S} J$ Fitzgerald, ${ }^{1} \mathrm{M}$ Egan, ${ }^{2} \mathrm{R}$ Costello, ${ }^{1} \mathrm{R}$ Sheahan. ${ }^{1}$ Department of Cardiology, Beaumont Hospital, Dublin, Ireland; ${ }^{2}$ Department of Respiratory Medicine Beaumont Hospital, Dublin, Ireland

\subsection{6/heartjn|-2020-ICS.19}

Background Patients with Duchenne muscular dystrophy (DMD) typically exhibit cardiac dysfunction and arrhythmia. With increasing life expectancy due to advances in respiratory support, cardiomyopathy and associated dysrhythmia are fast becoming the primary cause of morbidity and mortality in this patient group. Despite advances, the correlation between genotype and cardiac phenotype remains poorly understood and individual registries small, with implementation of device therapy often delayed due to poor diagnostic image quality.

Methods A single-center registry for DMD patients was established and data including genotyping, medical therapy and investigations such as cardiac MRI, nt-Pro BNP levels, echocardiogram and holter monitor was analysed. The aim was to potentially identify predictors associated with a more severe cardiac phenotype.

Results A total of 22 patients (age 17 -31) with DMD were reviewed (demographics summarised in table 1). All patients were evaluated with echocardiography on at least one occasion (mean EF 44.3\%). Cardiac MRI was attempted in six patients, however due to contractures preventing access to the scanner only three were completed. 14 of 22 patients (64\%) demonstrated an impaired left ventricular ejection fraction $(\mathrm{EF})<50 \%$ (mean EF 41\%). Proximal 'hot spot' deletions/mutations (exon 2-19) appeared to be associated with a more pronounced reduction in EF - all those patients with proximal mutations demonstrated an EF < $45 \%$ (mean 41\%). Seven of the eight patients (87.5\%) with mutations involving $>1$ exon deletion demonstrated more severely impaired EF (mean EF $37.5 \%$ ) compared to those with single exon deletions (mean EF 52\%). Interestingly, one patient with a proximal mutation (exon 3-6 deletion) remains mobilising to distances up to $70 \mathrm{~m}$, however CMRI performed has shown a moderate degree of fibrosis with an EF of 42\%. Correlation between nt-Pro BNP levels and reduced EF (rEF) was not uniform, however a level $<100$

\begin{tabular}{|c|c|c|c|c|c|}
\hline & Age & Mutation & $\mathrm{EF}$ & nt-Pro BNP & $\begin{array}{l}\text { No. of HF } \\
\text { Medications }\end{array}$ \\
\hline । & 17 & Exon 3-6 Del & $42 \%$ & Not Available & 2 \\
\hline ॥ & 17 & Nonsense Exon 19 & $45 \%$ & Not Available & 2 \\
\hline III & 18 & Nonsense Exon 19 & $45 \%$ & Not Available & 2 \\
\hline IV & 19 & Exon 47-52 Del & $40 \%$ & 251 & 3 \\
\hline v & 20 & Exon 37-43 Del & $50 \%$ & 300 & 1 \\
\hline VI & 20 & Exon 45-53 Del & $30 \%$ & 37 & 3 \\
\hline VII & 17 & Exon 44-47 Del & $50 \%$ & Not Available & 3 \\
\hline VIII & 22 & Exon $45-50+52$ Del & $30 \%$ & 231 & 2 \\
\hline IX & 21 & Exon 43-53 Del & $25 \%$ & 37 & 3 \\
\hline$x$ & 21 & Exon 45 Del & $45 \%$ & 290 & 3 \\
\hline $\mathrm{XI}$ & 28 & Absent on IHC & $50 \%$ & 35 & 2 \\
\hline XII & 20 & Absent on IHC & $40 \%$ & 164 & 2 \\
\hline XIII & 31 & Uncertain & $20 \%$ & 108 & 2 \\
\hline XIV & 18 & Exon 68 Del & $45 \%$ & 140 & 3 \\
\hline$X V$ & 20 & Exon $68 \mathrm{Del}$ & $70 \%$ & 36 & 1 \\
\hline $\mathrm{XVI}$ & 20 & Exon 45 Del & $35 \%$ & 290 & 3 \\
\hline XVII & 19 & Exon $44 \mathrm{Del}$ & $55 \%$ & 12 & 2 \\
\hline XVIII & 26 & Uncertain & $55 \%$ & 24 & 1 \\
\hline XIX & 31 & Uncertain & $60 \%$ & 47 & 3 \\
\hline$x x$ & 17 & Uncertain & $55 \%$ & 113 & 3 \\
\hline XXI & 26 & Uncertain & $55 \%$ & Not Available & 1 \\
\hline XXII & 31 & Exon $50 \mathrm{Del}$ & $60 \%$ & 63 & 2 \\
\hline
\end{tabular}

Nt-Pro BNP - N-terminal pro-B-type natriuretic peptide, HF - Heart Failure, IHC - immunohistochemistry, EF - ejection fraction.

was associated with $\mathrm{EF}>55 \%$ in $89 \%$ of cases. Of those on steroid regimes, 6 (54\%) had impaired LVEF compared to $5(83 \%)$ of those not on steroid therapy. All patients are taking at least one class of heart failure modification, with $79 \%$ on two and $37 \%$ on three. Only one patient in the registry has had an ICD implanted. This patient has had a device for 10 years and in this time there have been no therapies delivered.

Conclusion Correlation between predictors and cardiac phenotype in a Duchenne population remains unreliable. Location and size of exon alteration appears to be indicative of more markedly impaired LV function, however larger studies are required to characterise this further and challenges remain with regard to accurate assessment of EF. The use of predictors in future may help to guide appropriate provision of device therapy.

\section{HOW DOES BMI INFLUENCE HEART FAILURE PROGRAMME OUTCOMES?-TESTING THE OBESITY PARADOX}

${ }^{1} \mathrm{~L}$ Byrne, ${ }^{1} \mathrm{P}$ Wheen, ${ }^{2} \mathrm{P}$ Murray, ${ }^{3} \mathrm{C}$ Minelli, ${ }^{1} \mathrm{D}$ O'Callaghan, ${ }^{1} \mathrm{C}$ Daly. ${ }^{1}$ St. James's Hospital, Dublin, Ireland; ${ }^{2}$ Tallaght University Hospital, Dublin, Ireland; ${ }^{3}$ MRHT

\subsection{6/heartjnl-2020-ICS.20}

Introduction A complex physiological relationship exists between obesity and heart failure (HF) with many large-scale studies reporting a paradoxical improvement in cardiovascular (CV) mortality in obese patients. Many of these studies retrospectively analyzed RCT trials not designed to investigate the role of obesity in HF outcomes. We aim to establish if 
different outcomes exist after HF programme completion based on body mass index (BMI).

Methods A multi-centre retrospective observational study was carried out in 3 hospitals' HF clinics. New patients referred to each HF service over a 12 month period were reviewed, or in a 24 month period in one centre, with a left ventricular ejection fraction (LVEF) $<50 \%$ were eligible for inclusion. Patient records were obtained to collect patient demographics, New York Heart Association (NYHA) class symptoms, NTproBNP, LVEF and medications at initial referral and at programme completion. Hospitalisation rate for $\mathrm{HF}$ and mortality was also noted. Patient characteristics and outcomes were compared between non-obese $(\mathrm{BMI}<29.9)$ and obese (BMI $>30)$.

Results 93 patients were included for analysis and a follow up of (mean $( \pm \mathrm{SD}) \quad 16.5$ months $( \pm 8.8)$ was obtained. 63 $(67.7 \%)$ had a BMI <29.9 and $30(32.3 \%)$ had a BMI $>30$. Obese patients were more likely to be male $(p=0.03)$, diabetic $(p=0.05)$ and have hypertension $(p<0.001)$. No significant differences in medical or device therapy was noted between groups. Obese patients had a greater improvement in their LVEF following programme completion $(10.5 \%$ ( \pm 11.7$)$ versus $7.95 \%( \pm 10.7))$, although this did not reach statistical significance $(p=0.34)$. Obese patients had significantly lower NT-pro BNP levels at HF programme completion ((median(IQR)) (546.4 () versus 962 (), $\mathrm{P}=0.02$ ) however this improvement was not significantly greater than that seen in non-obese patients $(p=0.8)$. Although no difference in mortality was noted between the groups (8 (12.7\%) versus 3 (10\%), $\mathrm{p}=0.73$ ), obese patients had significantly fewer HF hospitalisations than the non-obese group $(0.33( \pm 0.74)$ versus 0.04 $( \pm 0.2), p=0.005)$.

Conclusion Despite higher rates of diabetes and hypertension in obese patients, there was no significant difference in mortality, LVEF improvement and NT-proBNP improvement following HF programme completion between the 2 groups. However, obese patients had significantly less HF hospitalisations during the study period than non-obese patients.

\section{INFECTIVE ENDOCARDITIS POST-TRANSCATHETER AORTIC VALVE IMPLANTATION: EXPERIENCE FROM AN IRISH TERTIARY REFERRAL CENTRE}

${ }^{1} \mathrm{~A}$ Buckley, ${ }^{1} \mathrm{~B}$ Armstrong, ${ }^{1} \mathrm{R}$ Tanner, ${ }^{2} \mathrm{~S}$ Hassan, ${ }^{2} \mathrm{~B}$ Moran, ${ }^{1} \mathrm{~J}$ Byrne, 'S Groarke, ${ }^{2}$ R Margey, ${ }^{1,2}$ I Casserly. 'Mater Misericordiae University Hospital, Dublin, Ireland; ${ }^{2}$ Mater Private Hospital, Dublin, Ireland

\subsection{6/heartjnl-2020-ICS.21}

Background Prior studies of infective endocarditis (IE) posttranscatheter aortic valve implantation (TAVI) have reported an incidence of $1-2 \%$, a median time from TAVI to presentation with IE of $\sim 1$ year, and 1-year mortality rates of 40$50 \%$. In addition, a higher rate of enterococci as the culprit microorganism compared to staphylococci and streptococci has been reported.

Objectives To determine the clinical characteristics, microorganism spectrum, and outcomes of IE post-TAVI in an Irish tertiary referral centre.

Methods Using a prospective TAVI database, all patients diagnosed with IE post-TAVI were identified. Pre-defined baseline demographic, procedural and follow-up data were analysed. Specific additional retrospective analysis of medical records

\begin{tabular}{ll} 
Abstract 21 Table 1 & Culprit Micro-organism \\
\hline Culprit microorganism & $\mathrm{N}(\%)$ \\
\hline Streptococci & $7(41.1)$ \\
Strep mitis & $2(11.7)$ \\
Strep salivarius & $2(11.7)$ \\
Strep infantarius & $1(5.8)$ \\
Strep gordonii & $1(5.8)$ \\
Abiotrophia Defectiva & $1(5.8)$ \\
Enterococcus faecalis & $3(17.6)$ \\
Staphylococci & $3(17.6)$ \\
Staph aureus & $1(5.8)$ \\
Coagulase-negative staph & $2(11.7)$ \\
Culture Negative & $4(23.5)$ \\
\hline
\end{tabular}

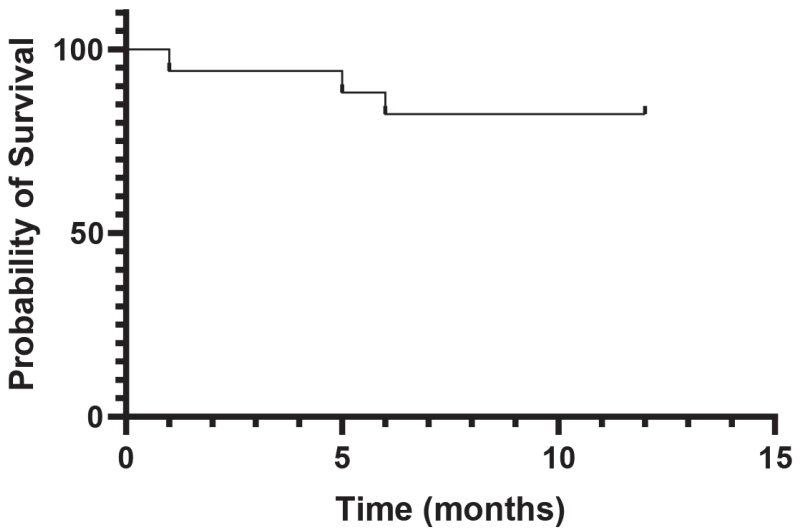

\begin{tabular}{|c|c|c|c|c|c|}
\hline Time (months) & 0 & 3 & 6 & 9 & 12 \\
\hline Number at risk & 17 & 16 & 14 & 14 & 14 \\
\hline
\end{tabular}

Survival probability estimate at one year is $0.82(95 \% \mathrm{Cl}=0.55-0.95)$

Abstract 21 Figure 1 Survival curve at 1 year for IE post-TAVI

were performed to assess the culprit microorganism and treatments for IE.

Results A total of 707 patients underwent TAVI during the study period. During a mean follow-up of 2.3 years, 17 (2.4\%) patients with IE were identified. According to the modified Duke criteria, IE was defined as definite and probable in $6(35 \%)$ and $11(65 \%)$ cases, respectively. The mean age was $78.7( \pm 13.7)$ years and $12(71 \%)$ were male. The median time from TAVI to presentation with IE was 7 months (IQR 5-13). The dominant culprit microorganisms identified were streptococci $(41.1 \%)$, with a low frequency of enterococcus faecalis $(17.6 \%)$ (table 1). Among the 17 patients with IE, there was one (6\%) major stroke and two patients (12\%) required surgical aortic valve replacement. There was one death during the initial hospitalization for IE. The KaplanMeier estimate of survival at 1 year was 0.82 (95\% CI = 0.55-0.95) (figure 1).

Conclusions This Irish TAVI cohort exhibited a similar incidence of IE post-TAVI and similar time to first presentation compared to prior international registries. There was a much lower rate of enterococcus compared to staphylococci and streptococci as the culprit microorganism. The 1-year mortality rate in this series was much lower than previously reported. 